\title{
Les "déflatés" du développement. De la tradition de dépendance à l'autogestion
}

\section{Edjenguèlè Mbonji}

\section{OpenEdition}

\section{Journals}

Édition électronique

URL : http://journals.openedition.org/apad/455

DOI : 10.4000/apad.455

ISSN : 1950-6929

Éditeur

LIT Verlag

Édition imprimée

Date de publication : 15 décembre 1999

\section{Référence électronique}

Edjenguèlè Mbonji, « Les "déflatés" du développement. De la tradition de dépendance à l'autogestion », Bulletin de l'APAD [En ligne], 18 | 1999, mis en ligne le 24 juillet 2006, consulté le 08 septembre 2020. URL : http://journals.openedition.org/apad/455 ; DOI : https://doi.org/10.4000/apad.455

Ce document a été généré automatiquement le 8 septembre 2020

Bulletin de l'APAD 


\title{
Les "déflatés" du développement. De la tradition de dépendance à l'autogestion
}

\author{
Edjenguèlè Mbonji
}

1 Au Cameroun, le mot "déflaté" définit l'agent de la fonction publique et du secteur parapublic qui est "compressé" selon une politique officielle de dégraissage des effectifs de l'Etat et de restrictions budgétaires drastriques qui ont suivi la grave crise économique et financière de 1986-1987. Acculé à passer sous les fourches caudines des mesures de redressement préconisées par les bailleurs de fonds, l'Etat camerounais n'eut d'autre recours que d'adopter une politique de rigueur économique et financière saupoudrée d'un mince volet social dont participe le phénomène des "déflatés", c'est-à-dire des employés dont on se sépare pour faire bonne mesure auprès du Fonds monétaire international et de la Banque mondiale et à qui on donne une certaine somme d'argent qualifiée de "prime d'encouragement à l'auto-emploi". En d'autres termes, en perdant son emploi dans la fonction publique, le "déflaté" doit devenir lui-même créateur d'emplois. Cette politique a-t-elle porté des fruits? Qui sont les "déflatés" et avaient-ils les moyens de leurs rêves? S'appuyant sur les données de l'évolution économique des dix dernières années, les comptes rendus d'évaluation de quelques projets initiés par des "déflatés", l'observation de la vie familiale des "compressés" et les journées de travail des agents du service public, les réponses à ces questions et à d'autres constituent les axes de notre réflexion. Celle-ci s'articule autour des thèmes suivants: l'environnement économique qui "prépare à l'avènement du déflaté", la posture mentale de l'agent public "déshabituant" à la prise d'initiative, le milieu de l'entreprenariat comme cadre normatif des attitudes vis-à-vis du risque et de la compétition, le paradigme développementaliste d'une technologie et d'une productivité au service de l'homme.

L'environnement socio-économique et l'occurrence du "déflaté"

2 Pour une meilleure intelligence du phénomène du "déflaté", il convient d'évoquer le marasme économique qui l'a préparé et dont il découle. A côté de la crise économique 
de 1986-1987, il faut signaler la double baisse de salaires des agents de la fonction publique en 1993, la dévaluation du franc CFA en 1994, l'irrégularité des salaires avec des mois élastiques de 45 à 60 jours, le blocage des avancements indiciaires et des changements de grades, le gel des recrutements dans la fonction publique, l'arrêt des concours administratifs, la non perception des pensions de retraite. Dans cette configuration, l'Etat fut appelé au dégraissage de ses effectifs que les partenaires financiers internationaux trouvaient pléthoriques, ceux-ci devant passer de 190.000 environ à 150.000 , et à la réduction de sa masse salariale.

3 La politique de compression ou de déflation, loin d'apparaître comme un lâchage pur et simple d'une partie des camerounais, fut alors conçue comme un moyen de résorber le chômage ambiant dont le taux dépassait les 50\%. Ainsi naît le "déflaté", agent de l'Etat mis au chômage avec une petite somme d'argent pour s'installer à son propre compte, s'inscrivant ainsi dans le train de mesures structurelles, législatives et réglementaires mises sur pied pour encourager les initiatives de création de nouveaux emplois salariés et indépendants. Comme pour donner raison à Jean Rivoli (1978:99) quand il reconnait à l'Etat la capacité de résoudre les problèmes au fur et à mesure qu'ils se présentent, l'Etat camerounais donne le ton, avec la création d'une part, du Fonds national de l'emploi (FNB) qui depuis 1990, a financé deux mille micro-projets, facilité la formation professionnelle de sept mille chômeurs et casé vingt-deux mille personnes dans des entreprises ; d'autre part, du Programme social d'urgence (PSU) instauré en 1994, et qui comptabilise huit mille chômeurs insérés sur le marché du travail, précisément dans le domaine de l'assainissement des grandes villes du Cameroun. Avec par ailleurs le projet Jeunes promoteurs, l'Etat entendait par ces initiatives de financement et d'encouragement des travaux à haute intensité de main-d'œuvre, s'attaquer à la gangrène du chômage et promouvoir l'auto-emploi. Dans cette logique, le "déflaté" est un futur chômeur par rapport à son emploi de la fonction publique, mais un chômeur invité à passer du statut de salarié à celui d'entrepreneur.

Le phénomène "déflaté"

4 Des critères définis par les experts de l'Etat, il ressort que la catégorie de "déflaté" regrouperait les "retraitables" et les sureffectifs identifiés à partir de la notion de poste de travail, c'est-à-dire selon le rôle, la fonction précise et nécessaire que tout agent de l'Etat assume dans le but $\mathrm{du}$ fonctionnement rationnel de l'organigramme administratif. Tout agent dont la position ne peut être impartie à une place dans cette architecture est candidat au dégraissage.

5 A ces critères d'ancienneté et d'opérationnalité, l'on a pu ajouter les cas d'agents dont le dossier disciplinaire préparait à la radiation. Par acquis de conscience, l'Etat inclut dans un second temps la notion de "départ volontaire", c'est-à-dire la catégorie de fonctionnaires et agents de l'Etat désireux de rompre eux-mêmes leur contrat avec l'Etat et d'utiliser le pécule promis pour se convertir dans les affaires. Aguichés par cette perspective, il y eut de la part des candidats une véritable ruée vers l'or au point que la masse des volontaires souhaitant quitter la fonction publique suscita l'appréhension de l'Etat de ne plus disposer du minimum de personnel nécessaire à son fonctionnement. La pratique de la déflation a ainsi été suspendue jusqu'à présent, d'autant que les résultats escomptés par le gouvernement n'étaient pas ceux visibles sur le terrain.

6 Au début de l'opération "déflaté", si l'on a pu noter une frustration certaine chez les agents de l'Etat désignés selon les critères gouvernementaux pour être licenciés, c'est 
parce que les "déflatés" de cette catégorie avaient l'impression de faire l'objet d'une sanction. Mais ils se ravisèrent après avoir pris acte de la situation sociale, financière et économique du pays, situation faite de compressions et de dépôts de bilan dans le privé et le parapublic - malgré les restructurations, les contrats de performance -, et dans la fonction publique, de retard des salaires, de blocage des avancements, de baisse des rémunérations et de non perception des pensions de vieillesse. A peu de chose près, c'est la même analyse que firent les candidats au départ volontaire qui appliquèrent le principe de sens commun "un tiens vaut mieux que deux tu auras".

7 De ce point de vue, "déflatés volontaires" et "déflatés imposés" acceptèrent de bonne grâce la somme accompagnant leur départ comme une bouée de sauvetage. De la part du gouvernement, il s'agissait simplement de répondre aux exigences du volet social du Plan d'ajustement structurel (PAS) en permettant à ses anciens employés de s'investir dans l'auto-emploi.

8 Cependant, l'on peut à juste titre s'interroger sur la signification que les uns et les autres donnaient à ce pécule d'encouragement à la création d'emploi. En outre, il n'est pas inopportun de se demander si les conditions sociologiques, législatives et structurelles étaient les meilleures pour transformer un employé en employeur, un salarié en investisseur. Avant de répondre à ces questions, relevons l'ancienneté de la pratique de l'auto-emploi parmi de nombreux fonctionnaires et agents de l'Etat au Cameroun où le fonctionnaire a souvent pu être à la fois salarié et entrepreneur. En effet, il est courant de rencontrer dans la même personne de l'employé de l'Etat en Afrique le phénomène de la concomitance des métiers, c'est-à-dire un cumul de multiples professions. Ainsi, l'agent de l'Etat émargeant au budget public, peut faire dans le même temps des affaires privées, en livrant par exemple du matériel de bureau dans divers ministères, ou en entretenant une ou deux plantations, comme l'y autorise la loi. Si pour certains, il s'agit d'un adjuvant ou d'un traitement d'appoint pour arrondir des fins de mois de plus en plus difficiles, il faut dire que pour d'autres "cumulards" des fonctions, l'attrait des affaires et des retombées financières sont telles qu'ils deviennent de véritables opérateurs économiques ayant pignon sur rue au détriment du bon fonctionnement de la machine de l'Etat dont ils font toujours partie. Lorsque l'on jette un coup d'œil sur les "déflatés" de la fonction publique, force est de constater qu'ils appartiennent presque tous non pas à la catégorie des "cumulards" qui ont réussi dans les affaires, mais plutôt à celle des "débrouillards" c'est-à-dire ces fonctionnaires moyens ou du bas de l'échelle professionnelle qui ont trouvé dans la pratique des fonctions multiples l'unique moyen de survivre dans un environnement de pauvreté financière où, malgré sa modicité et son irrégularité, le salaire versé par l'Etat restait le bienvenu.

9 Dans un cas comme dans l'autre, la pratique de la concomitance des métiers a précédé mais également renvoie à la politique de l'auto-emploi qu'adoptera l'Etat acculé au dégraissage du trop-plein de ses effectifs. Compte tenu de la similarité qu'il est possible d'établir entre la pratique des activités privées et l'incitation à l'auto-emploi dans le cadre de la "dimension sociale" des Plans d'ajustement structurel consentis par le gouvernement camerounais, on se serait attendu à voir les fonctionnaires "déflatés" dans de bonnes dispositions spirituelles et techniques de création et d'implantation d'initiatives de développement. Or la réaction première des "déflatés" a été la frustration: faut-il le rappeler, ceux qui avaient réussi la synthèse entre fonction publique et activité privée n'étaient pas nombreux et, malgré l'engouement ultérieur 
dû à la prise en compte de la situation économique du pays, la frustration susmentionnée aura des répercussions sur la reconversion du salarié en entrepreneur.

Car être "déflaté" c'est perdre son emploi, fut-il de rémunération modeste; c'est également perdre sa dignité de chef de famille, c'est être soi-même dévalué parce que désormais incapable de subvenir aux besoins de la famille. Les autorités camerounaises ne s'y sont pas trompées en utilisant le mot déflation qui, par opposition à inflation c'est-à-dire augmentation, hausse, montée, signifie diminution, baisse, etc. ; le "déflaté" se sent donc un être diminué, rabaissé. Les conséquences sociologiques de cet état de choses restent encore à étudier dans la société camerounaise où des ménages furent brisés, l'avenir de la progéniture compromis, cela dans un environnement où la plupart des sociétés d'Etat mettaient la clé sous la porte et où les entreprises privées fermaient ; tout cela induisant un taux de chômage de 50\%. Certes, le "déflaté" est un chômeur provisoire puisqu'il a l'espoir de se reconstruire ou de se réinsérer dans le tissu économique; mais il peut aussi devenir chômeur définitif si son initiative d'auto-emploi échoue. Pour expliquer l'installation du "déflaté" dans un état de chômage prolongé, certains économistes parlent du "mirage du chèque".

Le mirage du chèque

11 Le dernier chèque de la déflation n'est pas perçu dans sa réalité de rupture du cordon ombilical avec l'Etat, ou plutôt est-il trop bien perçu dans la mesure où il suscite angoisse et esprit festif: angoisse, parce que dernier salaire devant résoudre par anticipation tous les problèmes de loyer, de ration, de scolarité sans oublier les sollicitations de la famille élargie. Parce qu'accompagné de la fameuse prime d'encouragement à l'auto-emploi, le dernier chèque peut sembler consistant et par conséquent, inspirer une véritable folie dépensière s'exprimant par l'achat de la voiture dont on a toujours rêvé, l'accomplissement du voyage qu'on a toujours envisagé, etc. Cette véritable revanche sur soi ou sur les riches que sous-tend le désir de rattraper le temps perdu doit s'analyser pour ce qu'il est par rapport au problème du chômage, c'est-à-dire l'insouciance des lendemains ; car si l'argent reçu permet au "déflaté" une socialisation rétrospective lui permettant de se déployer dans des comportements de la classe aisée, ce déploiement éphémère vers les valeurs élitistes et les emblèmes de la réussite est appelé à prendre fin par un réveil brutal à la rudesse du quotidien. Le pécule alloué, de prime abord intéressant, ne l'était que par allusion au traitement habituel.

Les difficultés de l'auto-emploi

Elles sont de plusieurs ordres: structurel, réglementaire, économique et financier. Disons simplement que beaucoup de "déflatés" qui ont voulu s'investir dans les prestations de service ou le commerce, ont dû affronter une kyrielle de formalités dont ils n'avaient pas nécessairement l'habitude; ainsi, pour prendre un exemple dans le domaine des transports, mettre un taxi en circulation exige la constitution des documents suivants: patente, assurance, vignette, carte grise, carte bleue, visite technique, stationnement, formulaire de tarifs (donné par les syndicats de transporteurs). Or, la confection de ces divers documents est du ressort de plusieurs administrations et la connaissance des services mis à contribution ici n'est pas à la portée du premier venu.

13 A ces difficultés d'ordre administratif, il faut ajouter celles dues à l'absence d'expertise dans la gestion d'une structure de développement, aussi petite soit-elle. Gérer une affaire demande des compétences dans les domaines administratif, financier, 
technique, commercial et comptable, et ce qui caractérise le "déflaté" est justement la confusion de ces divers registres dans la conduite de son initiative d'emploi ; car ne l'oublions pas, le "déflaté" est devenu employeur sans préparation et il n'est pas rare que son emploi étant devenu son unique source de revenus, l'argent versé le soir par le chauffeur de taxi soit investi le lendemain dans le paiement de la scolarité ou bien, que la recette journalière de l'échoppe ne serve au paiement de la facture d'eau ou d'électricité.

Par ailleurs, il n'est pas inutile de s'intéresser à la personne du "déflaté" et aux moyens mis à sa disposition pour l'auto-emploi. Un coup d'œil sur les 6.000 "déflatés" du public donne à voir que nombre d'entre eux appartenaient aux catégories les moins diplômées et les plus défavorisées financièrement. Leur déflation du budget de l'Etat allait-elle les transformer en génies des affaires? D'autre part, le montant des indemnités de compression étant fonction du traitement salarial, c'est fort logiquement que les bas salaires ont générés de basses indemnités : le "déflaté" le moins nanti a reçu 600.000 Francs CFA, tandis que le plus payé a perçu 19 millions. C'est également en toute logique que leur départ n'a pas eu de réelle incidence sur la baisse de la masse salariale, peut-être aussi parce que les effectifs de la fonction publique étaient gonflés par une catégorie de personnel "fantôme" qui n'existait que sur le papier et qui pourtant émargeait bel et bien au budget public; il a fallu un certain nombre d'opérations d'assainissement du fichier de l'Etat (Opération Antilope, Recensement général avec présence effective) pour les déceler.

En réalité, les "déflatés" furent compressés sans grand effet sur la réduction du nombre d'agents de l'Etat et le rééquilibrage du budget puisqu'aujourd'hui, les recrutements ont repris dans les secteurs de la santé, de la défense, de l'éducation, de la justice. L'on pourrait penser que si l'auto-emploi dans les centres urbains nécessitait une procédure administrative et une expertise dont le "déflaté" n'avait pas la pratique, il lui aurait été plus aisé de s'investir dans l'agriculture en créant une plantation; et certains l'ont d'ailleurs fait et furent en butte aux difficultés liées à toute mise sur pied d'une activité tournant le dos aux méthodes artisanales et "empiriques". Ainsi, l'acquisition d'un terrain de petite ou moyenne superficie se heurte aux considérations foncières "traditionnelles" où la gestion du patrimoine obéit à une logique patriarcale selon laquelle l'acquisition d'un terrain doit respecter la primogéniture. De ce point de vue, il n'est pas facile au premier venu de se retrouver propriétaire d'une grande surface cultivable sauf à débourser une forte somme dont ne disposait pas forcément le "déflaté". A côté du problème foncier, s'élèvent ceux liés aux pépinières, aux engrais, à la main-d'œuvre, au transport et à la commercialisation qui n'ont pas rendu la tâche facile aux nouveaux entrepreneurs.

Et même dans le cas de certaines expériences plus structurées et organisées parce que bénéficiant de la technicité des structures préposées au courtage en développement, à l'exemple des experts du Fonds national de l'emploi (FNE), du Fonds d'aide et de garantie aux petites et moyennes entreprises (FOGAPE), à ce jour, l'évaluation finale des diverses initiatives révèle un constat d'échec. Pourtant avons-nous dit, parmi les "déflatés" se trouvent des fonctionnaires ayant pratiqué la concomitance des métiers du temps de leur présence dans la fonction publique. Pourquoi donc échouent-ils dès lors qu'on leur demande de s'investir à temps plein dans l'auto-emploi ? Qu'est devenue leur expérience passée dans le domaine entrepreneurial ?

Le "fonctionnaire affairiste" 
17 Pour la mise en route de son affaire, le fonctionnaire "cumulard" avait juste besoin d'une inscription au registre de commerce avec un numéro statistique, d'une patente et d'un impôt libératoire. Nous sommes là loin de la composition complète d'un dossier de création d'une micro-entreprise. Ses prestations de service se réduisaient à la livraison du matériel de bureau et à l'entretien des bâtiments administratifs. Or il est apparu que dans la plupart des cas, il s'agissait en réalité de livraisons fictives, de fausses factures sans oublier les pratiques de surfacturation. Les analyses consacrées à ce monde des affaires avec l'Etat montrent la complexité des démarches à suivre, les tracasseries administratives dont le corollaire est la corruption des fonctionnaires, la pression fiscale, le tout faisant du système une "pieuvre budgétivore" servant plus à enrichir les serviteurs de l'Etat qu'à œuvrer au bon fonctionnement de la machine administrative. Il en découle que le "fonctionnaire affairiste" n'apprend pas réellement à maîtriser les rouages du milieu des affaires, habitué qu'il est à contourner les règles du jeu officielles au profit de raccourcis dans l'acquisition de l'argent facile, raccourcis que sont la corruption, le détournement, la non déclaration des revenus, le changement perpétuel d'adresse.

18 Il va s'en dire qu'un opérateur économique amateur de pareilles gymnastiques ne peut se prévaloir de quelque expérience licite et "rationnelle" des affaires. Aussi le "déflaté" encouragé à l'auto-emploi, se distinguera-t-il par son inexpérience et une non culture de l'emploi ; s'il faut rendre justice à ceux des agents du service public et parapublic qui ont su tirer leur épingle du jeu en réussissant à s'imposer dans le monde des affaires, il faut également convenir qu'une bonne frange de ces agents ne pratiquait les métiers parallèles que comme adjuvant, en complément de leur salaire dans un contexte social de pauvreté financière où les besoins à satisfaire étaient plus nombreux que les moyens qu'accorde une grille de salaires à la limite de l'indécence.

Le "déflaté" et l'auto-emploi : un échec prévisible

19 Autant que nous le sachions et de l'avis des experts gouvernementaux et des institutions internationales, la politique officielle de déflation n'a pas débouché sur les résultats escomptés. Le fichier du personnel de l'Etat a certes été nettoyé et la facture salariale de l'Etat dégrossie, mais la reprise des recrutements dans quelques ministères montre que le surpeuplement de l'administration correspondait aussi à une mauvaise utilisation des ressources humaines. La déflation fut également un échec dans la mesure où les "déflatés" dans leur majorité sont allés gonfler les rangs des chômeurs, rangs déjà massifs en raison des licenciements du privé et du parapublic en faillite ou en restructuration. Il est tout à fait surprenant que le gouvernement camerounais n'ait pas fait faire d'études approfondies sur le sort de ces premières victimes de la crise économique de 1987 et de l'entrée au Fonds monétaire international quelques années plus tard. Sans doute acculés à des choix draconiens et restrictifs par les institutions de Bretton Woods, les décideurs camerounais n'ont pas pris le recul nécessaire pour concevoir une véritable politique de réinsertion des compressés dans les circuits économiques au travers d'une incitation rationnellement organisée à l'auto-emploi.

En outre, il faut signaler l'absence totale d'une structure spéciale d'accueil, d'orientation, d'encadrement et de suivi des "déflatés" en dehors du Fonds national de l'emploi au demeurant largement débordé par l'ampleur de sa mission qui est de s'occuper des chômeurs "en tout genre". Ses quelques commis préposés au conseil des "déflatés" se contentaient d'indications purement formelles, sans réelle possibilité d'encadrement pratique et de suivi ultérieur. 
21 A côté de l'insuffisance, voire de l'inexistence des structures de prise en charge des fonctions d'intermédiaires du développement, il importe de relever la quantité de "paperasserie" et une procédure chaotique à "effet de flipper" propres à décourager toute velléité entrepreneuriale. Tout opérateur économique sait combien il est difficile d'aller de ministère en ministère pour la constitution d'un dossier de création d'une micro-entreprise. Conscient de la complexité de la procédure, le gouvernement entend initier la révision du Code des investissements. Il est également question du principe du "guichet unique" devant permettre l'obtention dans un même espace de toutes les pièces afférentes à l'ouverture d'un dossier de création d'emploi.

L'échec de l'impréparation et de la dépendance

Les avis autorisés s'accordent à reconnaître que les "déflatés" mis en situation de s'investir dans l'auto-emploi manquaient cruellement de préparation. On ne s'improvise pas employeur ou entrepreneur après de longues années passées dans le rôle de salarié de la fonction publique. La sociologie du travail a établi des corrélations négatives entre le rendement dans une structure de haute productivité, où les employés sont soumis à une certaine rigueur, et les structures étatiques africaines où se reproduisent des logiques politiques clientélistes et ethniques génératrices de comportements "permissifs" dans les services. La "liberté" des agents de l'Etat, l'absence de contrainte horaire, l'idée camerounaise selon laquelle le travail du ngomna 1 ne finit jamais, en d'autres termes qu'il faut prendre tout son temps dans l'accomplissement des tâches administratives, ne manquent pas d'engendrer des réflexes de passivité, de lenteur, de non productivité difficiles à corriger dans la mesure où ils n'ont aucune incidence sur l'échéance mensuelle du salaire.

De ce point de vue, l'observation des comportements des agents publics montre moins un attachement au travail qu'une dépendance, une soumission à la régularité des salaires dont l'avènement n'est lié à aucun effort particulier à fournir. Pourquoi se gênerait-on? L'absence d'une mystique du travail au profit de l'attente frénétique des fins de mois peut s'analyser par ailleurs comme la recherche de l'argent facile. Quand bien même l'agent de l'Etat aurait développé des stratégies d'emploi parallèles pour compenser la modicité de ses revenus, il lui est difficile de se départir de la dépendance du salaire. Il n'y a qu'à voir le désarroi qui s'empare des fonctionnaires à l'annonce des retards dans le paiement de leurs salaires.

Sorti de cette ambiance et devant donc "se prendre en charge", il faut à l'agent de l'Etat déconstruire ses anciens repères, rompre avec son statut d'assisté de l'Etat et formuler de nouvelles perspectives, de nouvelles attentes, de nouveaux comportements, une "néo-culture" dont parlent Paul-Marc Henry et Basile Kossou (1985 : 31). Le moins que l'on puisse dire est qu'il semble malaisé de désapprendre des attitudes acquises après deux ou trois décennies au service de l'Etat.

Certes, ce qui définit l'homme est sa capacité d'adaptation et il est admis que tout ce qui est de l'ordre du social peut s'apprendre, mais le "déflaté" a-t-il eu le temps d'acquérir l'esprit d'entreprise, d'apprendre les normes liées à l'auto-emploi, de remplacer la mentalité de salarié subalterne par celle de décideur, de créateur ? Si l'on risque un parallèle entre la gestion négro-africaine du temps de travail et de l'effort dans les domaines de la chasse, la pêche, l'agriculture de subsistance, la poterie ou la forge d'une part, et dans la fonction publique d'autre part, on est frappé par la similarité de rythme et de non contrainte, sinon celle des calendriers imposés par le temps ou les intempéries, par la même logique de satisfaction du minimum et de non 
préoccupation d'une l'accumulation vue comme excédentaire: d'un côté on travaille juste ce qu'il faut pour aujourd'hui puisque l'on reviendra demain, de l'autre, on satisfait aux besoins du jour sans grand souci de ceux du lendemain, puisqu'on a le temps d'y remédier dès leur occurrence. Nous sommes là aux antipodes de J'univers concurrentiel du secteur privé où règne le principe "time is money" et où l'on ne compte ni son temps, ni sa peine.

La logique d'un modèle

Le "déflaté" dans le sillage de l'auto-développement ou de l'auto-emploi, sur le mode individuel ou associatif, est l'aboutissement logique d'un modèle organisationnel articulé plus sur la recherche du bien-être de l'homme que sur celle du profit. Dans la fonction publique ou sur la base de l'auto-emploi, quelle est la place réelle du "déflaté" dans un système de production dont les enjeux le plus souvent lui échappent? Il est dans la nature du procès de productivité et de rentabilité de produire des résidus et de s'en délester, comme il est dans celle de l'école de sécréter des échecs ou dans celle du développement d'engendrer le mal-développement et le sous-développement, véritable "tourniquet de l'absurde" (Schwarz 1980 : 127) marqué par le gaspillage des matières premières, un endettement astronomique, l'abandon des cultures vivrières le dépérissement de l'agriculture, la clochardisation des villes, une fracture sociale ostracisant les misérables. Que tout ceci prenne la forme des licenciements pour cause de privatisation ou de délocalisation en Occident, ou celle de la figure du "déflaté" au Cameroun ne change rien au résultat final, à savoir l'inégalité des sorts. La prise en compte de cette inégalité s'exprime ailleurs par l'existence d'une politique de solidarité nationale et de sécurité sociale organisée. Dans bien des pays africains au sud du Sahara, beaucoup reste à faire pour les exclus du développement.

L'étude du quotidien et du devenir des "déflatés" est un champ ouvert, et cette réflexion, qui ne visait pas à l'exhaustivité concernant ce phénomène social et économique complexe, s'est attelée plus modestement à indiquer qu'un séjour prolongé à l'ombre de la fonction publique entraîne l'intériorisation de toute une culture de routine, de dépendance et d'attente du salaire qui ne prépare pas à l'auto-prise en charge innovatrice et initiatrice de solutions nouvelles. Si le "déflaté" est apparu comme l'échec d'une politique officielle, d'organisation et de gestion des services de l'Etat, il porte aussi en lui une part de responsabilité au travers de sa "soumission" à des mécanismes aliénant sa créativité. Il ne s'agit pas ici de faire le procès de tel ou tel modèle de productivité, mais de montrer qu'il existe une corrélation entre un modèle d'organisation du travail et des formes d'inventions sociales soutenues par un corps de normes précis, de montrer la difficulté de la transition d'un lieu de déploiement de comportements assujettis à une finalité hétéro-déterminée vers un espace concurrentiel d'autogestion, et également de montrer la vanité du "mythe de la lutte contre la pauvreté par les projets de productivité" (Michaïlof 1984 : 29). Alors peut-être faudrait-il aller au delà du "développement négocié" selon l'expression apadienne (Bierschenk \& Le Meur 1997) pour repenser les rapports entre cultures et développement (Mbonji 1988) sous un angle endogène et humain. 


\section{BIBLIOGRAPHIE}

Bierschenk, T. \& Le Meur P.-Y. (dir.) 1997. "Le Développement négocié : courtiers, savoirs, technologies". Bulletin de l'APAD 12 (cahier thématique), Hamburg, Lit Verlag.

Gosselin, G. 1980. L'Afrique désenchantée - Théorie et politique du développement. Paris : Anthropos.

Gouvernement du Cameroun, 1998. Déclaration de stratégie de lutte contre la pauvreté. Yaoundé : Déclaration gouvernementale.

Henry, P.-M. \& Kossou B. 1985. La dimension culturelle du développement. Lomé : Nouvelles Editions Africaines.

Kabou, A. 1991. Et si l'Afrique refusait le développement. Paris : L'Harmattan.

Mbonji, E. 1988. Les cultures de développement en Afrique. Yaoundé : Osiris-Africa.

Michaïlof, S. 1984. Les apprentis-sorciers du Développement. Paris : Economica.

Rivoli, J. 1978. Le budget de l'Etat. Paris : Editions du Seuil.

Schwarz, A. (dir.) 1980. Les faux prophètes de l'Afrique ou de l'africanisme. Laval (Québec) : Presse de l'Université.

\section{NOTES}

1.Ngomna : expression camerounaise dérivant de la déformation de gouvernement.

\section{AUTEUR}

\section{EDJENGUÈLÈ MBONJI}

Département de sociologie et anthropologie. Université de Yaoundé I 